$\xi_{p}=-$

\title{
Is dark energy - an alias of cosmic rotational kinetic energy?
}

\author{
U. V.S. Seshavatharam ${ }^{1}$, S. Lakshminarayana ${ }^{2}$ \\ ${ }^{1}$ Honorary faculty, I-SERVE, Alakapuri, Hyderabad-35, Telangana, India \\ ${ }^{2}$ Dept. of Nuclear Physics, Andhra University, Visakhapatnam-03, AP, India \\ *Corresponding author E-mail:seshavatharam.uvs@gmail.com
}

\begin{abstract}
Considering Planck scale and Mach's principle, theoretically it is possible to show that magnitude of the currently believed dark energy is equal to the magnitude of current cosmic rotational kinetic energy.
\end{abstract}

Keywords: Planck Scale; Mach's Principle; Dark Energy; Rotational Kinetic Energy; Cosmic Age; Cosmic Scale Factor.

\section{Introduction}

We suppose that, the observable universe is an evolving and rotating sphere about the point of big bang and Planck scale is the characteristic energy scale associated with big bang and quantum gravity [1]. We define the Planck scale Hubble parameter as $\mathrm{H}_{\mathrm{pl}} \cong \sqrt{\mathrm{c}^{5} / \mathrm{G} \hbar}$ and apply it to cosmological data fitting in the form of $\left[1+\ln \left(\mathrm{H}_{\mathrm{pl}} / \mathrm{H}_{\mathrm{t}}\right)\right]^{\mathrm{n}}$ where $\mathrm{H}_{\mathrm{t}}$ is the running Hubble parameter and $\mathrm{n}$ is a suitable power index.

To have a closed and massive universe, we choose 'Mach's principle'. In this context, one of our assumption, $\left(\mathrm{GM}_{0} / \mathrm{R}_{0} \mathrm{c}^{2}\right) \cong 1$ can be given some consideration at fundamantal level. One can find interesting technical discussion on this assumption by D.W.Sciama, R.H. Dicke, C. Brans and G. J. Whitrow [2-4].

We review the currently believed phenomena of 'inflation' [5], [6] 'acceleration' and 'dark energy' [7], [8], [9] with increasing super luminal expansion speeds and increasing super luminal rotational speeds.

\section{Workable assumptions connected with Planck scale}

With the following simple and logical assumptions, most of the currently believed cosmological observations can be reviewed and refined at fundamental level.

1) With reference to big bang and Planck scale, Hubble parameter associated with Planck scale can be defined as $\mathrm{H}_{\mathrm{pl}} \cong \sqrt{\mathrm{c}^{5} / \mathrm{G} \hbar} \cong 1.8549215 \times 10^{43} \mathrm{sec}^{-1}$

2) Speed of light can be considered as the initial cosmic expansion speed.

3) At any stage of cosmic evolution, from and about the point of big bang, a) $t \cong\left[1+\ln \left(H_{t} / H_{0}\right)^{2}\right]\left(\frac{1}{H_{t}}\right)$ can be considered as the cosmic age. It may be noted that, for the current case, $H_{t} \cong H_{0}$ and $t_{0} \cong\left[1+\ln \left(H_{t} / H_{0}\right)^{2}\right]\left(\frac{1}{H_{t}}\right) \cong\left(\frac{1}{H_{0}}\right)$.

b) $\frac{G M_{t}}{R_{t} c^{2}} \cong 1$ where $\mathrm{M}_{\mathrm{t}}$ is the cosmic mass and $\mathrm{R}_{\mathrm{t}}$ is the cosmic radius or distance travelled.

c) Magnitude of cosmic angular velocity $\omega_{t}$ is equal to $\mathrm{H}_{\mathrm{t}}$.

d) $\mathrm{V}_{\mathrm{t}} \cong \sqrt{1+\ln \left(\mathrm{H}_{\mathrm{pl}} / \mathrm{H}_{\mathrm{t}}\right)} \times \mathrm{c}$ can be considered as the cosmic expansion speed.

e) Average distance travelled, $S_{t} \cong\left(\frac{V_{t}+c}{2}\right) t$ can be considered as the cosmic radius $R_{t}$.

f) Ratio of critical energy density and thermal energy density is equal to $\left[1+\ln \left(\mathrm{H}_{\mathrm{pl}} / \mathrm{H}_{\mathrm{t}}\right)\right]^{2}$.

\section{Discussion on the proposed assumptions and their consequences or results}

We would like to highlight the following points.

1) Modern cosmologists strongly believe that current universe is acceelrating. But they are silent in quantifying the past and current cosmic expansion speeds. In general, 'cosmic acceleration' means, 'rate of increase' in cosmic expansion speed. Based on assumptuion 3d, we treied our level best in quantifying the past and current cosmic expansion speeds.

2) Even though, modern cosmology is strongly believing in current cosmic acceleration, it is silent in quantifying the current cosmic acceleraton. Along with the assumed cosmic age, we assume the current and initial cosmic expansion speeds and thereby estimating the past and current cosmic radii. It is very clear that, current cosmic expansion speed is slowly increasing with increasing cosmic age. Based on this 
fact, we can have the following possible observations.

a) With increasing cosmic age, current universe is expanding with a slowly increasing speed of $11.885 \mathrm{c}$.

b) Magnitude of the future cosmic expansion speed depends on the magnitude of the future Hubble parameter.

c) By knowing the time to time future cosmic temperatures, from relation (1), corresponding future hubble parameters can be estimated and thus, corresponding future cosmic expansion speeds can be estimated.

3) With reference to the proposed assumptions, current universe seems to constitute 267 Hubble spheres. According to Mihran Vardanyan et al [10], the curvature scale of the Universe is conservatively constrained to be $R_{c}>42 \mathrm{Gpc}$ (99\%), corresponding to a lower limit to the number of Hubble spheres in the Universe NU > 251 (99\%). This coincidence clearly indicates the workability of our proposed assumptions.

4) We consider continuous super luminal expansions and hence it is possible to understand the currently observed large scale cosmic homogeniety or isotropy. Thus the 'inflation' concept can be relinquished [5,6]. In addition, concepts associated with 'fine tuning' of 'beginning of inflation' can also be ignored. Important point to be noted is that, modern estimate of cosmic radius is strictly assumed to obey 'inflation' whereas our estimate of cosmic radius (that is twice of modern estiamte) is independent of 'inflation'.

5) As the observed universe is very large and observers cannot reach all parts of the univese, one may be forced to believe in 'temperature isotropy'. In reality, as universe is continuously assumed to be expanding at increasing super luminal speeds, expecting 'temperature isotropy' may not be reasonable.

6) Since it is assumed that, universe is always expanding with increasing super luminal speeds, generally believed 'Lambda term ' can be ignored in our proposed model.

7) Without a radial in-flow of matter in all directions towards one specific point, one cannot expect a big crunch and without a big crunch, one cannot expect a big bang. Really if there was a 'big bang' in the past, with reference to formation of big bang as predicted by GTR and with reference to the cosmic rate of expansion that might had taken place simultaneously in all directions at a 'naturally selected rate' about the point of big bang - 'point' of big bang can be considered as the characteristic reference point of cosmic expansion in all directions. Thinking in this way, to some extent, point of big bang can be considered as a possible center of cosmic expansion.

8) It may be noted that, many cosmologists are working on 'cosmic rotation' [11-52]. According to Fani Dosopoulou et al [11]: "Current observations are consistent with small amounts of universal rotation, which means that, if the universe rotates, it does so very slowly. This is also in agreement with the inflationary scenario, where the exponential expansion is expected to essentially eliminate any traces of primordial vorticity. Nevertheless, most (if not all) astrophysical structures rotate, which raises the question whether their rotation is of cosmological origin, or a relatively recent addition due to local physical processes. Magnetic fields have long been known to act as sources of rotational distortions and the agent responsible for their generation is the field's tension. Consequently, one could argue that the origin of cosmic magnetism and that of universal rotation are closely (if not directly) related. Put another way, magnetized universes should also rotate".

9) From modern estimates, cosmic radius about earth is 46.5 billion light years and from our estimate, cosmic radius about the point of big bang is 90 billion light years and ratio of these two radii is very close to $1 / 2$. Estimated radii point of view, factor $1 / 2$ is not a big issue. As earth is far away from the cosmic boundary, even though, at present, if universe is really rotating with very small angular velocity, one may not be able to observe the effects of cosmic rotation from and about earth.

10) Considering all the proposed assumptions collectively, it is certainly possible to show that, ratio of currently believed 'dark enery density' and proposed 'rotational kinetic energy density' is equal to unity. This coincidence casts doubt on the existence of 'dark energy' at fundamanetal level and needs further study. It may be noted that, very recently, Naser Mostaghel, in his paper [53] clearly demonstrated an alternative way of describing the expansion of space without involving the controversial dark energy.

11) Even though our proposed model is independent of galactic redshifts, galactic distances and galactic receding speeds, with proposed assumptions, outline of the currently believed evolving cosmic structure can be understood very easily. By measuring the actual galactic receding speeds, assumption $3 \mathrm{~d}$ can be investigated further.

12) In any model of cosmology, fundamental questions to be solved are: 1) why do 'dark matter' and 'visible matter' have their measured values totaling to $\sim 33 \%$ of critical energy? 2) Why do 'dark energy' has its measured values of $\sim 68 \%$ of critical energy? 3) How to estimate their past and future magnitudes? These are the puzzling questions raised by the Royal Swedish Academy of Sciences [7] in 2011. In the conclusion part, Royal Swedish Academy of Sciences say: "The study of distant supernovae constitutes a crucial contribution to cosmology. Together with galaxy clustering and the CMB anisotropy measurements, it allows precise determination of cosmological parameters. The observations present us with a challenge, however: What is the source of the dark energy that drives the accelerating expansion of the Universe? Or is our understanding of gravity as described by general relativity insufficient? Or was Einstein's "mistake" of introducing the cosmological constant one more stroke of his genius? Many new experimental efforts are underway to help shed light on these questions". In this context, here it is worth to mention that, according to Serkan Zorba, 'dark energy' and 'dark matter' both are inertial effects [54], [55]. In section-4, we tried our level best in answering these basic questions with assumption $3 \mathrm{~b}$ which is having a long history in General relativity and Cosmology connected with 'inertia and gravity'.

13) Believing in the existence of 'dark matter', stability of galactic clusters, galactic rotation speeds, oscillating patterns observed in CMB power spectrum, glaring inconsistency observed in Bullet cluster and patterns observed in galactic structural evolution can be understood. Important point to be noted is that, by estimating the current cosmic mass density and rotational kinetic energy, current dark matter percentage can be estimated accurately. See the proposed relations (16) and (17) in section-4.

14) We are working on applying our assumptions to review and understand the (big bang) nucleosynthesis. Based on the new approach and obtained results, scope and workability of our assumptions can be scrutinized and validated.

\section{Various applications of $\left[1+\ln \left(\mathrm{H}_{\mathrm{pl}} / \mathrm{H}_{0}\right)\right]$ in cosmology}

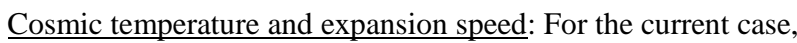

$\mathrm{aT}_{0}^{4} \cong\left[1+\ln \left(\frac{\mathrm{H}_{\mathrm{pl}}}{\mathrm{H}_{0}}\right)\right]^{-2}\left(\frac{3 \mathrm{H}_{0}^{2} \mathrm{c}^{2}}{8 \pi \mathrm{G}}\right) \cong\left(\frac{\mathrm{c}}{\mathrm{V}_{0}}\right)^{4}\left(\frac{3 \mathrm{H}_{0}^{2} \mathrm{c}^{2}}{8 \pi \mathrm{G}}\right)$

where $V_{0}$ is the current cosmic expansion speed. 


$$
\begin{aligned}
& T_{0} \cong\left[1+\ln \left(\frac{H_{p l}}{H_{0}}\right)\right]^{-1 / 2}\left(\frac{3 H_{0}^{2} c^{2}}{8 \pi G a}\right)^{\frac{1}{4}} \cong\left(\frac{c}{V_{0}}\right)\left(\frac{3 H_{0}^{2} c^{2}}{8 \pi G a}\right)^{\frac{1}{4}} \\
& \sqrt{\frac{3 \mathrm{H}_{0}^{2} \mathrm{c}^{2}}{8 \pi \mathrm{GaT}_{0}^{4}} \cong\left[1+\ln \left(\frac{\mathrm{H}_{\mathrm{pl}}}{\mathrm{H}_{0}}\right)\right] \cong\left(\frac{\mathrm{V}_{0}}{\mathrm{c}}\right)^{2}}
\end{aligned}
$$

With trial-error, it is noticed that, if $\mathrm{H}_{0} \cong 70.0 \mathrm{~km} / \mathrm{sec} / \mathrm{Mpc}$, obtained $T_{0} \cong 2.7208 \mathrm{~K}$. This estimated value of $H_{0}$ seems to lie in between the values recommended by references [8] and [9]. Clearly speaking, $\left\{(67.7 \pm 0.66)>H_{0}<(73.24 \pm 1.74)\right\} \mathrm{km} / \mathrm{sec} / \mathrm{Mpc}$.

$\sqrt{1+\ln \left(\frac{H_{p l}}{H_{0}}\right)} \cong\left(\frac{V_{0}}{c}\right) \cong 11.8851$

and $V_{0} \cong 3.563063 \times 10^{9} \mathrm{~m} \cdot \mathrm{sec}^{-1}$

Cosmic radius: For the current case, cosmic radius or distance travelled,

$$
\begin{aligned}
& \mathrm{S}_{0} \cong \mathrm{R}_{0} \cong\left(\frac{\mathrm{V}_{0}+\mathrm{c}}{2}\right) \mathrm{t}_{0} \cong\left(\frac{\mathrm{c}}{2 \mathrm{H}_{0}}\right)\left[1+\sqrt{1+\ln \left(\frac{\mathrm{H}_{\mathrm{pl}}}{\mathrm{H}_{0}}\right)}\right] \\
& \cong 6.4425\left(\frac{\mathrm{c}}{\mathrm{H}_{0}}\right) \cong 8.514 \times 10^{26} \mathrm{~m}
\end{aligned}
$$

From our estimate, current distance (observable and nonobservable) about the point of big bang is $90 \mathrm{Gly}=27.6 \mathrm{Gpc}$. Our estimate seems to be approximately 1.935 times higher than modern estimation. Clearly speaking, current universe seems to constitute 267 Hubble spheres [10].

Galactic receding speeds: Based on relation (4), within the current radius of $90 \mathrm{Gly}=27.6 \mathrm{Gpc}$, from and about the point of big bang, galactic receding speeds can be approximated with the following relation.

$$
\mathrm{v}_{\mathrm{g}} \cong\left(\frac{\mathrm{d}_{\mathrm{g}}}{\mathrm{S}_{0}}\right) \mathrm{V}_{0} \cong\left(\frac{\mathrm{d}_{\mathrm{g}}}{\mathrm{S}_{0}}\right) 11.8851 \mathrm{c} \cong\left\{\frac{2 \sqrt{1+\ln \left(\mathrm{H}_{\mathrm{pl}} / \mathrm{H}_{0}\right)}}{\left[1+\sqrt{1+\ln \left(\mathrm{H}_{\mathrm{pl}} / \mathrm{H}_{0}\right)}\right]}\right\}\left(\mathrm{d}_{\mathrm{g}} \mathrm{H}_{0}\right)
$$

where $d_{g}$ is the current galactic distance from the point of big bang and $v_{g}$ is the current galactic receding speed. Based on this relation (5), within the current boundary of $90 \mathrm{Gly}=27.6 \mathrm{Gpc}$, galactic distances corresponding to assumed galactic receding speeds can be expressed in the following way.

$$
\mathrm{d}_{\mathrm{g}} \cong\left(\frac{\mathrm{v}_{\mathrm{g}}}{\mathrm{v}_{0}}\right) \mathrm{S}_{0} \cong\left(\frac{\mathrm{v}_{\mathrm{g}}}{11.8851 \mathrm{c}}\right) 8.514 \times 10^{26} \mathrm{~m}
$$

By co-relating the estimated galactic distances and actual receding speeds with observed galactic red shifts, further research can be carried out.

Cosmic mass and mass density: Based on relation (4), current visual and non-visual cosmic radius is around $90 \mathrm{Gly}=27.6 \mathrm{Gpc}$. With reference to assumption $3 \mathrm{~b}$, current mass of our (visible and invisible parts) universe can be estimated with the following relation.

$$
\begin{aligned}
& \mathrm{M}_{0} \cong \frac{\mathrm{c}^{2} \mathrm{R}_{0}}{\mathrm{G}} \cong 1.14654 \times 10^{54} \mathrm{~kg} \\
& \text { where, } \mathrm{R}_{0} \cong \frac{\mathrm{V}_{0}+\mathrm{c}}{2 \mathrm{H}_{0}} \cong\left(\frac{\mathrm{c}}{2 \mathrm{H}_{0}}\right)\left[1+\sqrt{1+\ln \left(\frac{\mathrm{H}_{\mathrm{pl}}}{\mathrm{H}_{0}}\right)}\right] .
\end{aligned}
$$

With this estimated mass, current cosmic mass density can be expressed with the following relation. $\left(\rho_{\text {mass }}\right)_{0} \cong \frac{3 \mathrm{c}^{2}}{4 \pi \mathrm{GR}_{0}^{2}} \cong\left[1+\sqrt{1+\ln \left(\frac{\mathrm{H}_{\mathrm{pl}}}{\mathrm{H}_{0}}\right)}\right]^{-2} \frac{3 \mathrm{H}_{0}^{2}}{\pi \mathrm{G}}$

$$
\cong 4.43505 \times 10^{-28} \mathrm{~kg} \cdot \mathrm{m}^{-3}
$$

Now ratio of mass energy density and critical energy density [8] can be expressed with the following relation.

$\left(\frac{\rho_{\text {mass }} \mathrm{c}^{2}}{\rho_{\text {critical }} \mathrm{c}^{2}}\right)_{0} \cong 8\left[1+\sqrt{1+\ln \left(\frac{\mathrm{H}_{\mathrm{pl}}}{\mathrm{H}_{0}}\right)}\right]^{-2} \cong 0.048185$

Cosmic rotational kinetic energy: From assumptions $3 \mathrm{a}, 3 \mathrm{~b}$ and $3 \mathrm{c}$, current cosmic rotational energy can be estimated in the following way.

$$
\left(\mathrm{K}_{\text {rot }}\right)_{0} \cong \frac{1}{2} \mathrm{I}_{0} \omega_{0}^{2} \cong \frac{1}{2} \mathrm{I}_{0} \mathrm{H}_{0}^{2}
$$

As current 'mass density' is very small in magnitude, current observable universe can be considered as a thin spherical shell and hence its corresponding current moment of inertia can be expressed with the following relation.

$\mathrm{I}_{0} \cong \frac{2}{3} \mathrm{M}_{0} \mathrm{R}_{0}^{2}$

From the above two relations, current cosmic rotational kinetic energy can be expressed with the following simple relation.

$\left(\mathrm{K}_{\text {rot }}\right)_{0} \cong \frac{1}{3} \mathrm{M}_{0} \mathrm{R}_{0}^{2} \omega_{0}^{2} \cong \frac{1}{3} \mathrm{M}_{0} \mathrm{R}_{0}^{2} \mathrm{H}_{0}^{2} \cong 1.4257 \times 10^{72} \mathrm{~J}$

Surprisingly it is noticed that, ratio of proposed current cosmic rotational energy density and currently believed dark energy density is very close to unity. It can be expressed in the following way.

$\left[\frac{\left(\mathrm{K}_{\mathrm{rot}}\right)_{0}}{(4 \pi / 3) \mathrm{R}_{0}^{3}}\right] /\left[0.68 \times\left(\frac{3 \mathrm{H}_{0}^{2} \mathrm{c}^{2}}{8 \pi \mathrm{G}}\right)\right] \cong 0.98$

where $0.68 \times\left(\frac{3 \mathrm{H}_{0}^{2} \mathrm{c}^{2}}{8 \pi \mathrm{G}}\right)$ is the currently believed dark energy densi-

ty [8]. With reference to critical energy density, current rotational kinetic energy density can be expressed with the following relation.

$$
\frac{\left(\mathrm{K}_{\text {rot }}\right)_{0}}{(4 \pi / 3) \mathrm{R}_{0}^{3}} \cong \frac{\mathrm{M}_{0} \omega_{0}^{2}}{4 \pi \mathrm{R}_{0}} \cong \frac{\mathrm{M}_{0} \mathrm{H}_{0}^{2}}{4 \pi \mathrm{R}_{0}} \cong \frac{\mathrm{H}_{0}^{2} \mathrm{c}^{2}}{4 \pi \mathrm{G}}
$$

Clearly speaking, ratio of current cosmic rotational kinetic energy density and critical energy density is equal to $\frac{2}{3} \cong 0.666667$. It can be expressed in the following way.

$\left[\frac{\left(\mathrm{K}_{\mathrm{rot}}\right)_{0}}{(4 \pi / 3) \mathrm{R}_{0}^{3}}\right] /\left[\frac{3 \mathrm{H}_{0}^{2} \mathrm{c}^{2}}{8 \pi \mathrm{G}}\right] \cong \frac{2}{3}$

If one is willing to consider this coincidence as a 'heuristic coincidence', it is possible to say that, currently believed 'dark energy' is nothing but the current cosmic rotational kinetic energy. It is for further study.

Dark matter density: Based on the currently believed 'flat' model concept and current observations, 'dark matter' energy density can be fitted in the following way. 


$$
\begin{aligned}
& \left(\rho_{\text {d.matter }}\right)_{0} c^{2} \cong\left\{1-\left[\begin{array}{l}
\frac{\left(K_{\text {rot }}\right)_{0}}{(4 \pi / 3) R_{0}^{3}} \\
+\frac{M_{0} c^{2}}{(4 \pi / 3) R_{0}^{3}}
\end{array}\right]\right\}\left(\frac{3 H_{0}^{2} c^{2}}{8 \pi G}\right) \\
& \cong\left\{1-\left[\frac{2}{3}+0.048185\right]\right\}\left(\frac{3 H_{0}^{2} c^{2}}{8 \pi G}\right) \cong 0.28515\left(\frac{3 H_{0}^{2} c^{2}}{8 \pi G}\right)
\end{aligned}
$$

Qualitatively this can be compared with the currently believed 'dark matter' energy density [8] and needs further study. Proceeding further, we noticed a very interesting relation in the following way.

$\left(\rho_{\text {mass }}\right)_{0} c^{2} \cong \frac{\left[\left(\rho_{\text {mass }}\right)_{0} c^{2}+\left(\rho_{\text {d.matter }}\right)_{0} c^{2}\right]^{2}\left[\left(\rho_{\text {rot }}\right)_{0} c^{2}\right]^{2}}{\left[\left(\rho_{\text {critical }}\right)_{0} c^{2}\right]^{3}}$

where, $\left(\rho_{\text {rot }}\right)_{0} c^{2} \cong \frac{\left(\mathrm{K}_{\text {rot }}\right)_{0}}{(4 \pi / 3) \mathrm{R}_{0}^{3}} \cong\left(\frac{2}{3}\right)\left(\frac{3 \mathrm{H}_{0}^{2} \mathrm{c}^{2}}{8 \pi \mathrm{G}}\right)$. we are working on understanding this very strange and very simple relation. Based on this relation,

if $\left(\rho_{\text {mass }}\right)_{0} \cong 0.048185\left(\frac{3 H_{0}^{2}}{8 \pi G}\right)$,

obtained $\left(\rho_{\text {d.matter }}\right)_{0} \cong 0.2811\left(\frac{3 H_{0}^{2}}{8 \pi G}\right)$.

Cosmic age connected with $3000 \mathrm{~K}$ : According to standard cosmology, cosmic age corresponding to a temperature of $\sim 3000 \mathrm{~K}$ and redshift of $\sim 1100$ is around $\sim 3,80,000$ years. Based on our proposed assumptions and from relation

$H_{t} \cong 2.47323 \times 10^{-12} \mathrm{sec}^{-1}$, obtained $T_{t} \cong 2991.9 \mathrm{~K}$.

Corresponding cosmic age can be fitted with assumption $3 \mathrm{a}$ in the following way.

$$
\begin{aligned}
t_{T \approx 2992 K} & \cong\left[1+\ln \left(\frac{H_{T \approx 2992 K}}{H_{0}}\right)^{2}\right] \frac{1}{H_{T \approx 2992 K}} \\
& \cong 3,69,047 \text { years. }
\end{aligned}
$$

Cosmic scale factor connected with $3000 \mathrm{~K}$ : With reference to the data prepared as per the proposed relations, it is possible to show that,

$$
\left\{\left(\frac{a_{\text {now }}}{a_{\text {then }}} \cong(z+1)\right) \cong \frac{T_{t}}{T_{0}}\right\} \cong \frac{V_{0}}{V_{t}} \sqrt{\frac{H_{t}}{H_{0}} \cong \sqrt{\left(\frac{V_{0}^{2}}{H_{0}}\right) /\left(\frac{V_{t}^{2}}{H_{t}}\right)}}
$$

Where $V_{0}$ and $V_{t}$ represent the current and past cosmic expansion speeds respectively. Interesting point to be noted is that, magnitude of $\left(\frac{V_{t}^{2}}{H_{t}}\right)$ increases with cosmic age.

For the $\mathrm{CMBR}$ redshift case, i.e. $T_{t} \cong 3000 \mathrm{~K}$ and $z \cong 1100$

$$
H_{t} \cong 2.47323 \times 10^{-12} \mathrm{sec}^{-1} \cong H_{z \approx 1100 \cdots(\text { say })}
$$

Estimated, $T_{t} \cong 2991.9 \mathrm{~K} \approx T_{z} \approx 1100 \cdots . .($ say $)$
Estimated, $V_{t} \cong 3.3832 \times 10^{9} \mathrm{~m} \cdot \mathrm{sec}^{-1} \approx V_{z \approx 1100 \cdots . .(\text { say })}$

Estimated, $\frac{V_{t}^{2}}{H_{t}} \cong 4.62798 \times 10^{30} \mathrm{~m}^{2} \cdot \mathrm{sec}^{-1} \cong \frac{V_{z \approx 1100}^{2}}{H_{z \approx 1100}} \ldots . .($ say $)$

Estimated, $\frac{V_{0}^{2}}{H_{0}} \cong 5.59635 \times 10^{36} \mathrm{~m}^{2} \cdot \mathrm{sec}^{-1}$

It is noticed that,

$$
(z+1) \cong \sqrt{\left(\frac{V_{0}^{2}}{H_{0}}\right) /\left(\frac{V_{z \approx 1100}^{2}}{H_{z \approx 1100}}\right)} \cong 1099.6
$$

With further study,

1) $\left(\frac{V_{t}^{2}}{H_{t}}\right)$ can be replaced with $\left(\frac{V_{t}}{H_{t}}\right)$. If so, $(z+1) \cong 1071.5$

2) $(z+1)$ can be fitted with the following relation.

$$
(z+1) \approx\left(\frac{H_{t}}{H_{0}}\right)^{\frac{2}{3}}\left[1+\ln \left(\frac{H_{t}}{H_{0}}\right)^{2}\right]^{-2 / 3}
$$

\section{Conclusions}

By following the proposed assumptions, in this paper we tried our level best in estimating and co-relating the Hubble parameter, cosmic temperature, age, expansion speed, radius, mass, mass density, rotational kinetic energy, dark matter density and galactic receding speeds of the current expanding universe. Readers are strongly encouraged to see reference [56] for an elaborated early version of this short communication. Advantages of the proposed assumptions are:

1) Inherently connected with the Planck scale

2) Successful inplementaion of Mach's principle.

3) Logically very simple to implement and understand.

4) Resolves the key issues connected with currently believed 'inflation', 'cosmic horizon' and 'dark energy'.

5) Perfectly connects the current Hubble parameter and current cosmic temperature.

6) In all the cases, extrapolation to past and future is very easy.

7) With further research, a unified model of evolving quantum cosmology can be developed.

\section{Acknowledgements}

Authors are very much thankful to Dr. E. Terry Tatum, Dr. L.M. Chechin and Dr. Serkan Zorba for their kind and valuable technical discussion. One of the authors, Seshavatharam U.V.S, is indebted to professors K.V. Krishna Murthy, Chairman, Institute of Scientific Research in Vedas (I-SERVE), Hyderabad, India and Shri K.V.R.S. Murthy, former scientist IICT (CSIR), Govt. of India, Director, Research and Development, I-SERVE, for their valuable guidance and great support in developing this subject.

\section{References}

[1] Martin Bozowald. Quantum cosmology: a review. Rep. Prog. Phys. 78 (2015) 023901http://dx.doi.org/10.1088/0034-4885/78/2/023901.

[2] G. J. Whitrow. The Mass of the Universe. Nature 158, 165-166 (1946)http://dx.doi.org/10.1038/158165b0.

[3] D.W.Sciama. on the Origin of Inertia. MNRAS 113, 34, (1953) http://dx.doi.org/10.1093/mnras/113.1.34.

[4] C. Brans and R. H. Dicke. Mach's principle and a relativistic prin$\begin{array}{llll}\text { ciple of gravitation. Phys. Rev. 124, } 925 & 925\end{array}$ (1961)http://dx.doi.org/10.1103/PhysRev.124.925. 
[5] Guth AH. Inflationary universe: A possible solution to the horizon and flatness problems. Phys. Rev.; D23:347. (1981). http://dx.doi.org/10.1103/PhysRevD.23.347.

[6] Steinhardt PJ. The inflation debate: Is the theory at heart of modern cosmology deeply flawed? Scientific American; 304(4):18-25. (2011)http://dx.doi.org/10.1038/scientificamerican0411-36.

[7] The accelerating universe. Compiled by the Class for Physics of the Royal Swedish Academy of Sciences (2011). www.nobelprize.org/nobel_prizes/physics/laureates/2011/advanced -physicsprize2011.pdf

[8] Planck Collaboration: Planck 2015 Results. XIII. Cosmological Parameters.

[9] Adam G. Riess et al. A 2.4\% Determination of the Local Value of the Hubble Constant. Astrophys.J. 826 no.1. (2016)http://dx.doi.org/10.3847/0004-637X/826/1/56.

[10] MihranVardanyan et al. Applications of Bayesian model averaging to the curvature and size of the Universe. MNRAS Lett 413, 1, L91-L95 3933.2011.01040.x.

[11] L.M. Chechin. Rotation of the Universe at different cosmological epochs. Astron.Rep. 60 no.6, 535-541. (2016)http://dx.doi.org/10.1134/S1063772916040041.

[12] Demidchenko V. V. and Demidchenko V. I. The rotating universe. Liberal Arts in Russia. Vol. 5. No. 2. Pp. 131-160. (2016)http://dx.doi.org/10.15643/libartrus-2016.2.4

[13] Tatum, E.T., Seshavatharam, U.V.S. and Lakshminarayana, S. The Basics of Flat Space Cosmology. International Journal of Astronomy and Astrophysics, 5, 116-124 (2015)http://dx.doi.org/10.4236/ijaa.2015.52015.

[14] Tatum ET, Seshavatharam U.V.S, Lakshminarayana S. Flat space cosmology as a mathematical model of quantum gravity or quantum cosmology. International journal of astronomy and astrophysics. 5, 133-140. (2015)

[15] Tatum, E.T., Seshavatharam, U.V.S. and Lakshminarayana, S. Thermal Radiation Redshift in Flat Space Cosmology. Journal of Applied Physical Science International, 4, 18-26. (2015)

[16] Eugene Terry Tatum, U.V.S. Seshavatharam, and S. Lakshminarayana, Flat Space Cosmology as an Alternative to Lcdm Cosmology. Frontiers of Astronomy, Astrophysics and Cosmology, vol. 1, no. 2 (2015)

[17] U.V.S. Seshavatharam, S. Lakshminarayana. Friedmann cosmology: Reconsideration and New Results. International Journal of Astronomy, Astrophysics and Space Science, 1(2):16-26. (2014).

[18] Chechin, L.M. and Ibraimova, A.T. The Jeans Equation Generalization for the Rotating Universe. International Journal of Astronomy and Astrophysics, 4, 614-619 (2014)http://dx.doi.org/10.4236/ijaa.2014.44056.

[19] FaniDosopoulou and Christos G. Tsagas. Vorticity survival in magnetized Friedmann universes. Phys.Rev.D89:103519,(2014)http://dx.doi.org/10.1103/PhysRevD. 89.103519.

[20] Špringer J. Rotating and Expanding Universe in the Light of Flyby Anomaly and Path Distribution. GJSFR (A). volume 14, issue 2, 59-61.(2014).

[21] Chechin L.M. On the Modern Status of the Universe Rotation Problem. Journal of Modern Physics, 4, 126-132. (2013) http://dx.doi.org/10.4236/jmp.2013.48A012.

[22] Marcelo Samuel Berman, Fernando de Mello Gomide. Local and Global Stability of the Universe. Journal of Modern Physics, 4, 7-9, (2013)http://dx.doi.org/10.4236/jmp.2013.48A002.

[23] U. V. S. Seshavatharam .The Primordial Cosmic Black Hole and the Cosmic Axis of Evil. International Journal of Astronomy 1(2): 20-37, (2012)

[24] Sivaram C, KenathArun. Primordial Rotation of the Universe, Hydrodynamics, Vortices and Angular Momenta of Celestial Objects. The Open Astronomy Journal, 5, 7-11(2012) http://dx.doi.org/10.2174/1874381101205010007.

[25] Michael J. Longo, Detection of a Dipole in the Handedness of Spiral Galaxies with Redshifts z 0.04, Phys. Lett. B.699:224-229 (2011) http://dx.doi.org/10.1016/j.physletb.2011.04.008.

[26] WlodzimierzGodlowski. Global and local effects of rotation: $\mathrm{Ob}$ servational aspects. International Journal of Modern Physics D 20 1643 (2011)http://dx.doi.org/10.1142/S0218271811019475.

[27] U.V.S. Seshavatharam, Physics of Rotating and Expanding Black Hole Universe, Progress in Physics, vol. 2, pp7-14, (2010).

[28] Sidharth B.G. Is the Universe Rotating? Prespacetime Journal. Vol 1, Issue 7, pp. 1168-1173. (2010)

[29] Dmitri Rabounski. On the Speed of Rotation of Isotropic Space: Insight into the Redshift Problem. The Abraham Zelmanov Journal, Vol. 2, 208-223. (2009)
[30] Su S-C, Chu M-C. Is the universe rotating? Astrophysical Journal, 703 354. (2009).http://dx.doi.org/10.1088/0004-637X/703/1/354.

[31] Chapline G, et al. Tommy Gold Revisited: Why Does Not The Universe Rotate? AIP Conf.Proc.822:160-165,(2006). http://arxiv.org/abs/astro-ph/0509230.

[32] E. V. Kufshinova. and, V. F. Panov. Quantum Origin of a Rotating Universe of the Bianchi IX Type. Russian Physics Journal. Volume 48, Issue 6, pp 633-638 (2005)http://dx.doi.org/10.1007/s11182 005-0178-y.

[33] WlodzimierzGodlowski and MarekSzydlowsk. Dark energy and global rotation of the Universe. Gen.Rel.Grav. 35 21712187(2003)http://dx.doi.org/10.1023/A:1027301723533.

[34] Robert V Gentry. New Cosmic Center Universe Model Matches Eight of Big Bang's Major Predictions without the F-L Paradigm. CERN preprint, EXT-2003-022, (2003).

[35] Yuri N. Obukhov et al. Rotation in string cosmology. Class.Quant.Grav.20:1103-1114, (2003)http://dx.doi.org/10.1088/0264-9381/20/6/305

[36] Li-Xin Li. Effect of the Global Rotation of the Universe on the Formation of Galaxies. Gen.Rel.Grav. 30, 497(1998) http://dx.doi.org/10.1023/A:1018867011142.

[37] Rainer W. Kuhne. On the Cosmic Rotation Axis. Mod.Phys.Lett.A12:2473-2474 (1997)http://dx.doi.org/10.1142/S0217732397002594.

[38] Vladimir A. Korotky and Yuri N. Obukhov. On cosmic rotation. Gravity, Particles and Space-Time, eds. P. Pronin and G. Sardanashvily, pp. 421-439. (World Scientific: Singapore, 1996)

[39] Korotky, V.A. and Obukhov. Can cosmic rotation explain an apparently periodic universe? Y.N. Gen RelatGravit 26: 429. (1994)http://dx.doi.org/10.1007/BF02108047.

[40] Obukhov Y. N., Rotation in Cosmology, General Relativity and Gravitation 24, 121(1992) http://dx.doi.org/10.1007/BF00756780.

[41] Panov V. F., Sbytov, Yu. G., Accounting for Birch's observed anisotropy of the universe: cosmological rotation? Sov. Phys. JETP 74, 411(1992)

[42] G.Ruben. Cosmic rotation and the inertial system. Astrophysics and $\begin{array}{lll}\text { Space } & \text { Science } & \text { 177(1):465-470 }\end{array}$ http://dx.doi.org/10.1007/BF00643720.

[43] ÖYVIND GRÖN \& HARALD H. SOLENG. Decay of primordial cosmic rotation in inflationary cosmologies. Nature $328,501-503$ (1987)http://dx.doi.org/10.1038/328501a0.

[44] Barrow JD, Juszkiewicz R, Sonoda DH. Universal rotation - How large can it be? Mon. Not. R. Astron. Soc. 213: 917. (1985)http://dx.doi.org/10.1093/mnras/213.4.917.

[45] Valdes F., Tyson J. A., Jarvis J. F. Alignment of faint galaxy images: Cosmological distortion and rotation. Ap. J. 271, 431 (1983)http://dx.doi.org/10.1086/161210.

[46] Birch P. Is the universe rotating?, Nature 298, 451(1982)http://dx.doi.org/10.1038/298451a0.

[47] Novello M, Reboucas MJ. Rotating universe with successive causal and noncausal regions. Phys. Rev. D 19, 2850-2852 (1979) http://dx.doi.org/10.1103/PhysRevD.19.2850.

[48] F. M. Gomide. Cosmic Rotation and Mach's Principle in Three Special Cosmological Models. RevistaBrasileira de Física, Vol. 3 , No. 1, (1973)

[49] Hawking SW. On the rotation of the universe. Mon. Not. Royal. Astr. Soc. 142, 129- 141. http://dx.doi.org/10.1093/mnras/142.2.129.

[50] J.V. Narlikar. Newtonian Universes with Shear and Rotation $\begin{array}{llll}\text { MNRAS } & 126 & (2): & 203-208 .\end{array}$ http://dx.doi.org/10.1093/mnras/126.2.203.

[51] Kurt Godel. Rotating Universes in General Relativity Theory. Proceedings of the international Congress of Mathematicians in Cambridge, 1: 175-81, (1950)

[52] Gamov G., Rotating Universe? Nature 158, 549. (1946) http://dx.doi.org/10.1038/158549a0.

[53] Mostaghel, N. A New Solution for the Friedmann Equations. International Journal of Astronomy and Astrophysics, 6, 122-134 (2016)http://dx.doi.org/10.4236/ijaa.2016.61010.

[54] Serkan Zorba. A Modified FRW Metric to Explain the Cosmological Constant. Mod. Phys. Lett. A, 27, 1250106 (2012)http://dx.doi.org/10.1142/S0217732312501064.

[55] Serkan Zorba. Dark energy and dark matter as inertial effects. arXiv:1210.3021(2012)

[56] Seshavatharam, U. V. S. \& Lakshminarayana, S., Superluminal Expansion \& Rotation in Redshift Independent Accelerating Quantum Cosmology. Prespacetime Journal, Volume 7, Issue 11, pp. 1511 1534, (2016) 